\title{
Article \\ A New Algebraic Inequality and Some Applications in Submanifold Theory
}

\author{
Ion Mihai ${ }^{1, *,+}$ and Radu-Ioan Mihai ${ }^{2,+}+\mathbb{D}$ \\ 1 Department of Mathematics, University of Bucharest, 010014 Bucharest, Romania \\ 2 Faculty of Mathematics and Computer Science, University of Bucharest, 010014 Bucharest, Romania; \\ radu.mihai4@s.unibuc.ro \\ * Correspondence: imihai@fmi.unibuc.ro \\ + These authors contributed equally to this work.
}

check for

updates

Citation: Mihai, I.; Mihai, R.-I. A New Algebraic Inequality and Some Applications in Submanifold Theory. Mathematics 2021, 9, 1175. https:// doi.org/10.3390/math9111175

Academic Editor: Ana-Maria Acu

Received: 29 April 2021

Accepted: 19 May 2021

Published: 23 May 2021

Publisher's Note: MDPI stays neutral with regard to jurisdictional claims in published maps and institutional affiliations.

Copyright: (c) 2021 by the authors. Licensee MDPI, Basel, Switzerland. This article is an open access article distributed under the terms and conditions of the Creative Commons Attribution (CC BY) license (https:// creativecommons.org/licenses/by/ $4.0 /)$.

\begin{abstract}
We give a simple proof of the Chen inequality involving the Chen invariant $\delta(k)$ of submanifolds in Riemannian space forms. We derive Chen's first inequality and the Chen-Ricci inequality. Additionally, we establish a corresponding inequality for statistical submanifolds.
\end{abstract}

Keywords: Riemannian space form; submanifold; Chen invariants; Chen inequalities; statistical manifold; statistical submanifold

MSC: 53C40; 53C05

\section{Introduction}

One of the most important topics of research in the geometry of submanifolds in Riemanian manifolds is to establish sharp relationships between extrinsic and intrinsic invariants of a submanifold.

The most used intrinsic invariants are sectional curvature, scalar curvature and Ricci curvature. The main extrinsic invariant is the squared mean curvature.

There are well-known relationships between the above extrinsic and intrinsic invariants for a submanifold in a Riemannian space form: (generalized) Euler inequality, Chen-Ricci inequality, Wintgen inequality, etc.

In [1,2], B.-Y. Chen introduced a sequence of Riemannian invariants, which are known as Chen invariants. They are different in nature from the classical Riemannian invariants. B.-Y. Chen established optimal relationships between the squared mean curvature and Chen invariants for submanifolds in Riemannian space forms, known as Chen inequalities (see [2]). The proofs of these inequalities use an algebraic inequality, discovered by B.-Y. Chen in [1].

In the present paper, we give simple proofs of some Chen inequalities by using a different algebraic inequality.

Other Chen inequalities were proved in [3] by applying another inequality.

\section{Preliminaries}

The theory of Chen invariants and Chen inequalities was initiated by B.-Y. Chen $[1,2]$.

Let $(M, g)$ be an $n$-dimensional $(n \geq 2)$ Riemannian manifold, $\nabla$ its Levi-Civita connection and $R$ the Riemannian curvature tensor field on $M$. The sectional curvature $K(\pi)$ of the plane section $\pi \subset T_{p} M, p \in M$, is defined by

$$
K(\pi)=R\left(e_{1}, e_{2}, e_{1}, e_{2}\right)=g\left(R\left(e_{1}, e_{2}\right) e_{2}, e_{1}\right),
$$

where $\left\{e_{1}, e_{2}\right\}$ is an orthonormal basis of $\pi$. 
Let $\left\{e_{1}, \ldots, e_{n}\right\}$ be an orthonormal basis of $T_{p} M$. The scalar curvature $\tau$ at $p$ is given by

$$
\tau(p)=\sum_{1 \leq i<j \leq n} K\left(e_{i} \wedge e_{j}\right)
$$

where $K\left(e_{i} \wedge e_{j}\right)$ is the sectional curvature of the plane section spanned by $e_{i}$ and $e_{j}$.

If $X$ is a unit vector tangential to $M$ at $p$, consider the orthonormal basis $\left\{e_{1}=\right.$ $\left.X, e_{2}, \ldots, e_{n}\right\}$ of $T_{p} M$. The Ricci curvature is defined by

$$
\operatorname{Ric}(X)=\sum_{j=2}^{n} K\left(X \wedge e_{j}\right) \text {. }
$$

Let $L$ be an $r$-dimensional subspace of $T_{p} M$ and $\left\{e_{1}, \ldots, e_{r}\right\}$ an orthonormal basis of $L, 2 \leq r \leq n$. The the scalar curvature $\tau(L)$ of $L$ is given by $\tau(L)=\sum_{1 \leq \alpha<\beta \leq r} K\left(e_{\alpha} \wedge e_{\beta}\right)$.

In particular, for $r=2, \tau(L)$ is the sectional curvature of $L$ and for $r=n, \tau\left(T_{p} M\right)=$ $\tau(p)$ is the scalar curvature of $M$ at $p$.

B.-Y. Chen introduced a sequence of Riemannian invariants $\delta\left(n_{1}, \ldots, n_{l}\right)$, known as Chen invariants (see [2]).

The Chen first invariant is $\delta_{M}=\tau-\inf K$, where

$$
(\inf K)(p)=\inf \left\{K(\pi) \mid \pi \subset T_{p} M \text { plane section }\right\} .
$$

Let $l>0$ be an integer and $n_{1}, \ldots, n_{l} \geq 2$ integers such that $n_{1}<n$ and $n_{1}+\ldots+n_{l} \leq n$. The Chen invariant $\delta\left(n_{1}, \ldots, n_{l}\right)$ is defined by

$$
\delta\left(n_{1}, \ldots, n_{l}\right)(p)=\tau(p)-\inf \left\{\tau\left(L_{1}\right)+\ldots+\tau\left(L_{l}\right)\right\},
$$

where $L_{1}, \ldots, L_{l}$ are mutually orthogonal subspaces of $T_{p} M$ with $\operatorname{dim} L_{j}=n_{j}, j=1, \ldots, l$.

For $l=1$ in particular, one has $\delta(2)=\delta_{M}$ and $\delta(n-1)=$ max Ric, with

$$
\max \operatorname{Ric}(p)=\max \left\{\operatorname{Ric}(X) \mid X \in T_{p} M, g(X, X)=1\right\} .
$$

We shall consider the Chen invariant $\delta(k)$, which is given by

$$
\delta(k)(p)=\tau(p)-\inf \tau\left(L_{k}\right),
$$

where $L_{k}$ is any $k$-dimensional subspace of $T_{p} M$.

\section{An Algebraic Inequality}

In this section, we give an algebraic inequality and study its equality case. As an application, we get a simple proof of the Chen inequality for the invariant $\delta(k)$.

Lemma 1. Let $k, n$ be nonzero natural numbers, $2 \leq k \leq n-1$, and $a_{1}, a_{2}, \ldots, a_{n} \in \mathbb{R}$. Then

$$
\sum_{1 \leq i<j \leq n} a_{i} a_{j}-\sum_{1 \leq \alpha<\beta \leq k} a_{\alpha} a_{\beta} \leq \frac{n-k}{2(n-k+1)}\left(\sum_{i=1}^{n} a_{i}\right)^{2} .
$$

Moreover, the equality holds if and only if $\sum_{\alpha=1}^{k} a_{\alpha}=a_{j}$, for all $j \in\{k+1, \ldots, n\}$.

Proof. We prove this Lemma by using the Cauchy-Schwarz inequality. We have

$$
\left(\sum_{i=1}^{n} a_{i}\right)^{2}=\left(\sum_{\alpha=1}^{k} a_{\alpha}+a_{k+1}+\ldots+a_{n}\right)^{2} \leq
$$




$$
\begin{gathered}
\leq(n-k+1)\left[\left(\sum_{\alpha=1}^{k} a_{\alpha}\right)^{2}+a_{k+1}^{2}+\ldots+a_{n}^{2}\right]= \\
=(n-k+1)\left(\sum_{i=1}^{n} a_{i}^{2}+2 \sum_{1 \leq \alpha<\beta \leq k} a_{\alpha} a_{\beta}\right)= \\
=(n-k+1)\left[\left(\sum_{i=1}^{n} a_{i}\right)^{2}-2 \sum_{1 \leq i<j \leq n} a_{i} a_{j}+2 \sum_{1 \leq \alpha<\beta \leq k} a_{\alpha} a_{\beta}\right],
\end{gathered}
$$

which implies the desired inequality.

The equality holds if and only if we have equality in the Cauchy-Schwarz inequality, i.e., $\sum_{\alpha=1}^{k} a_{\alpha}=a_{j}$, for all $j \in\{k+1, \ldots, n\}$.

\section{Proof of the Chen Inequality for $\delta(K)$}

We apply Lemma 1 for obtaining a simple proof of the Chen inequality corresponding to the Chen invariant $\delta(k)$ for submanifolds in Riemannian space forms.

Let $\tilde{M}(c)$ be an $m$-dimensional Riemannian space form of constant sectional curvature $c$. The Euclidean space $\mathbb{E}^{m}$, the sphere $S^{m}$ and the hyperbolic space $H^{m}$ are the standard examples.

Consider $M$ an $n$-dimensional submanifold of $\tilde{M}(c)$ and denote by $h$ the second fundamental form of $M$ in $\tilde{M}(c)$. The mean curvature vector $H(p)$ at $p \in M$ is defined by

$$
H(p)=\frac{1}{n} \sum_{i=1}^{n} h\left(e_{i}, e_{i}\right)
$$

where $\left\{e_{1}, \ldots, e_{n}\right\}$ is an orthonormal basis of $T_{p} M$.

The submanifold $M$ is called minimal if the mean curvature vector $H(p)$ vanishes at any $p \in M$.

We recall the Gauss equation (see [4]):

$$
R(X, Y, Z, W)=c+g(h(X, Z), h(Y, W))-g(h(X, W), h(Y, Z)),
$$

for all vector fields $X, Y, Z, W$ tangential to $M$.

Theorem 1. Let $\tilde{M}(c)$ be an m-dimensional Riemannian space form of constant sectional curvature $c$ and $M$ an n-dimensional submanifold of $\tilde{M}(c)$. Then, for any $2 \leq k \leq n-1$, one has the following Chen inequality:

$$
\delta(k) \leq \frac{n^{2}(n-k)}{2(n-k+1)}\|H\|^{2}+\frac{1}{2}[n(n-1)-k(k-1)] c .
$$

Moreover, the equality holds at a point $p \in M$ if and only if there exist suitable orthonormal bases $\left\{e_{1}, \ldots, e_{n}\right\} \subset T_{p} M$ and $\left\{e_{n+1}, \ldots, e_{m}\right\} \subset T_{p}^{\perp} M$ such that the shape operators take the forms

$$
\begin{gathered}
A_{e_{n+1}}=\left(\begin{array}{cccccc}
a_{1} & \ldots & 0 & 0 & \ldots & 0 \\
\vdots & \ddots & \vdots & \vdots & & \vdots \\
0 & \ldots & a_{k} & 0 & \ldots & 0 \\
0 & \ldots & 0 & \mu & \ldots & 0 \\
\vdots & & \vdots & \vdots & \ddots & \vdots \\
0 & \ldots & 0 & 0 & \ldots & \mu
\end{array}\right), \quad \sum_{\alpha=1}^{k} a_{\alpha}=\mu, \\
A_{e_{r}}=\left(\begin{array}{cc}
A_{r} & 0 \\
0 & \mathbf{O}_{n-k}
\end{array}\right), \quad r=n+2, \ldots, m,
\end{gathered}
$$


where $A_{r}$ is a symmetric $k \times k$ matrix with trace $A_{r}=0$ and $\mathbf{O}_{n-k}$ is the $(n-k) \times(n-k)$ null matrix.

Proof. Let $p \in M, L \subset T_{p} M$ be a $k$-dimensional subspace and $\left\{e_{1}, \ldots, e_{k}\right\}$ be an orthonormal basis of $L$. We take $\left\{e_{1}, \ldots, e_{k}, e_{k+1}, \ldots, e_{n}\right\} \subset T_{p} M$ and $\left\{e_{n+1}, \ldots, e_{m}\right\} \subset T_{p}^{\perp} M$ as orthonormal bases, respectively.

Denote as usual by $h_{i j}^{r}=g\left(h\left(e_{i}, e_{j}\right), e_{r}\right), i, j=1, \ldots, n, r \in\{n+1, \ldots, m\}$, the components of the second fundamental form.

The Gauss equation implies

$$
\begin{aligned}
\tau & =\sum_{1 \leq i<j \leq n} K\left(e_{i} \wedge e_{j}\right)=\sum_{1 \leq i<j \leq n} R\left(e_{i}, e_{j}, e_{i}, e_{j}\right)= \\
& =\frac{n(n-1)}{2} c+\sum_{r=n+1}^{m} \sum_{1 \leq i<j \leq n}\left[h_{i i}^{r} h_{j j}^{r}-\left(h_{i j}^{r}\right)^{2}\right] .
\end{aligned}
$$

Additionally, by the Gauss equation one has

$$
\tau(L)=\frac{k(k-1)}{2} c+\sum_{r=n+1}^{m} \sum_{1 \leq \alpha<\beta \leq k}\left[h_{\alpha \alpha}^{r} h_{\beta \beta}^{r}-\left(h_{\alpha \beta}^{r}\right)^{2}\right] .
$$

Then we get

$$
\begin{gathered}
\tau-\tau(L)=\frac{1}{2}[n(n-1)-k(k-1)] c+ \\
+\sum_{r=n+1}^{m}\left(\sum_{1 \leq i<j \leq n} h_{i i}^{r} h_{j j}^{r}-\sum_{1 \leq \alpha<\beta \leq k} h_{\alpha \alpha}^{r} h_{\beta \beta}^{r}\right)-\sum_{r=n+1}^{m} \sum_{1 \leq i<j \leq n ;(i, j) \notin\{1, \ldots, k\}^{2}}\left(h_{i j}^{r}\right)^{2} .
\end{gathered}
$$

By using the algebraic inequality from the previous section, we obtain

$$
\begin{aligned}
\tau-\tau(L) \leq & \frac{n-k}{2(n-k+1)} \sum_{r=n+1}^{m}\left(\sum_{i=1}^{n} h_{i i}^{r}\right)^{2}+\frac{1}{2}[n(n-1)-k(k-1)] c= \\
& =\frac{n^{2}(n-k)}{2(n-k+1)}\|H\|^{2}+\frac{1}{2}[n(n-1)-k(k-1)] c
\end{aligned}
$$

which implies the inequality to prove.

If the equality case holds at a point $p \in M$, then we have equalities in all the inequalities in the proof, i.e.,

$$
\left\{\begin{array}{l}
\sum_{\alpha=1}^{k} h_{\alpha \alpha}^{r}=h_{j j}^{r}, \forall j \in\{k+1, \ldots, n\}, \\
h_{i j}^{r}=0, \forall 1 \leq i<j \leq n,(i, j) \notin\{1, \ldots, k\}^{2},
\end{array}\right.
$$

for any $r \in\{n+1, \ldots, m\}$.

If we choose $e_{n+1}$ parallel to $H(p)$, then the shape operators take the above forms.

Corollary 1. Let $\tilde{M}(c)$ be an m-dimensional Riemannian space form of constant sectional curvature $c$ and $M$ an n-dimensional submanifold of $\tilde{M}(c)$. If there exists a point $p \in M$ such that $\delta(k)(p)>\frac{1}{2}[n(n-1)-k(k-1)] c$, then $M$ is not minimal.

If $k=1$, we derive Chen's first inequality: 
Corollary 2. [1] Let $\tilde{M}(c)$ be an m-dimensional Riemannian space form of constant sectional curvature $c$ and $M$ an $n$-dimensional submanifold of $\tilde{M}(c)$. Then one has

$$
\inf K \geq \tau-\frac{n-2}{2}\left[\frac{n^{2}}{n-1}\|H\|^{2}+(n+1) c\right]
$$

Equality holds at a point $p \in M$ if and only if, with respect to suitable orthonormal bases $\left\{e_{1}, \ldots, e_{n}\right\} \subset T_{p} M$ and $\left\{e_{n+1}, \ldots, e_{m}\right\} \subset T_{p}^{\perp} M$, the shape operators take the following forms:

$$
\begin{gathered}
A_{e_{n+1}}=\left(\begin{array}{ccccc}
a & 0 & 0 & \ldots & 0 \\
0 & \mu-a & 0 & \ldots & 0 \\
0 & 0 & \mu & \ldots & 0 \\
\vdots & \vdots & \vdots & \ddots & \vdots \\
0 & 0 & 0 & \ldots & \mu
\end{array}\right), \\
A_{e_{r}}=\left(\begin{array}{ccccc}
h_{11}^{r} & h_{12}^{r} & 0 & \ldots & 0 \\
h_{12}^{r} & -h_{11}^{r} & 0 & \ldots & 0 \\
0 & 0 & 0 & \ldots & 0 \\
\vdots & \vdots & \vdots & \ddots & \vdots \\
0 & 0 & 0 & \ldots & 0
\end{array}\right), r=n+2, \ldots, m .
\end{gathered}
$$

Recall that $\delta(n-1)=\max$ Ric. Then, from Theorem 1 we deduce the Chen-Ricci inequality:

Corollary 3. [5] Let $\tilde{M}(c)$ be an m-dimensional Riemannian space form of constant sectional curvature $c$ and $M$ an $n$-dimensional submanifold of $\tilde{M}(c)$. Then, for any $p \in M$ and any unit vector $X$ tangential to $M$, one has

$$
\operatorname{Ric}(X) \leq \frac{n^{2}}{4}\|H\|^{2}+(n-1) c .
$$

We present the following examples:

Example 1. Let $k, n$ be integers such that $k \geq 2$ and $n \geq 2 k-1$. Consider the hypercylinder $M=S^{k} \times \mathbb{E}^{n-k} \subset \mathbb{E}^{n+1}$.

Clearly $\delta(k)=\tau=\frac{1}{2} k(k-1)$. Then the equality case of Theorem 1 holds identically if and only $n=2 k-1$, i.e., $M=S^{k} \times \mathbb{E}^{k-1}$.

Moreover, $\max$ Ric $=\frac{n^{2}}{4}\|H\|^{2}$ if and only if $k=2$ and $n=3$, i.e., $M=S^{2} \times \mathbb{E}$.

Example 2. The generalized Clifford torus.

Let $T=S^{k}\left(\sqrt{\frac{k}{n}}\right) \times S^{n-k}\left(\sqrt{\frac{n-k}{n}}\right) \subset S^{n+1} \subset \mathbb{E}^{n+2}, n>k \geq 1$.

It is known that $T$ is a minimal hypersurface of $S^{n+1}$, but a non-minimal submanifold of $\mathbb{E}^{n+2}$.

Obviously max Ric $=\max \left\{(k-1) \frac{n}{k},(n-k-1) \frac{n}{n-k}\right\}$.

Then $T \subset S^{n+1}$ does not satisfy the equality case of Theorem 1 for $\delta(n-1), \forall n \geq 2$.

If we consider $T \subset \mathbb{E}^{n+2}$, then it does not satisfy the equality case of Theorem 1 for $\delta(n-1), \forall n \geq 2$. 


\section{A Chen Inequality for Statistical Submanifolds}

A statistical manifold is an $m$-dimensional Riemannian manifold $(\tilde{M}, g)$ endowed with a pair of torsion-free affine connections $\tilde{\nabla}$ and $\tilde{\nabla}^{*}$, which satisfy

$$
Z g(X, Y)=g\left(\tilde{\nabla}_{Z} X, Y\right)+g\left(X, \tilde{\nabla}_{Z}^{*} Y\right)
$$

for any $X, Y, Z \in \Gamma(T \tilde{M})$. The connections $\tilde{\nabla}$ and $\tilde{\nabla}^{*}$ are called dual connections (see [6,7]), and it is easily seen that $\left(\tilde{\nabla}^{*}\right)^{*}=\tilde{\nabla}$. The pairing $(\tilde{\nabla}, g)$ is said to be a statistical structure. If $(\tilde{\nabla}, g)$ is a statistical structure on $\tilde{M}^{m}$, then $\left(\tilde{\nabla}^{*}, g\right)$ is a statistical structure too $[6,8]$.

Any torsion-free affine connection $\tilde{\nabla}$ on $\tilde{M}$ always has a dual connection given by

$$
\tilde{\nabla}+\tilde{\nabla}^{*}=2 \tilde{\nabla}^{0},
$$

where $\tilde{\nabla}^{0}$ is the Levi-Civita connection on $\tilde{M}$.

The dual connections are called conjugate connections in affine differential geometry (see [9]).

Denote by $\tilde{R}$ and $\tilde{R}^{*}$ the curvature tensor fields of $\tilde{\nabla}$ and $\tilde{\nabla}^{*}$, respectively. They satisfy

$$
g\left(\tilde{R}^{*}(X, Y) Z, W\right)=-g(Z, \tilde{R}(X, Y) W) .
$$

A statistical structure $(\tilde{\nabla}, g)$ is said to be of constant curvature $\varepsilon \in \mathbb{R}$ if

$$
\tilde{R}(X, Y) Z=\varepsilon\{g(Y, Z) X-g(X, Z) Y\} .
$$

A statistical structure $(\tilde{\nabla}, g)$ of constant curvature 0 is called a Hessian structure.

The Equation (2) implies that if $(\tilde{\nabla}, g)$ is a statistical structure of constant curvature $\varepsilon$, then $\left(\tilde{\nabla}^{*}, g\right)$ is also a statistical structure of constant curvature $\varepsilon$ (obviously, if $(\tilde{\nabla}, g)$ is Hessian, $\left(\tilde{\nabla}^{*}, g\right)$ is also Hessian).

The dual connections are not metric, then we cannot define a sectional curvature in the standard way. A sectional curvature on a statistical manifold was defined by $B$. Opozda [10].

More precisely, if one considers $p \in \tilde{M}, \pi$ a plane section in $T_{p} \tilde{M}$ and an orthonormal basis $\{X, Y\}$ of $\pi$, then a sectional curvature is defined by

$$
\tilde{K}(\pi)=\frac{1}{2}\left[g\left(\tilde{R}(X, Y) Y+\tilde{R}^{*}(X, Y) Y, X\right)\right],
$$

which is independent of the choice of the orthonormal basis.

Next, we consider a statistical manifold $(\tilde{M}, g)$ and a submanifold $M$ of dimension $n$ of $\tilde{M}$. Then $\left(M,\left.g\right|_{M}\right)$ is also a statistical manifold with the connection induced by $\tilde{\nabla}$ and induced metric $g$.

In Riemannian geometry, the fundamental equations are the Gauss and Weingarten formulae and the equations of Gauss, Codazzi and Ricci.

As usual, we denote by $\Gamma\left(T^{\perp} M\right)$ the set of the sections of the bundle normal to $M$.

In our case, for any $X, Y \in \Gamma(T M)$, according to [8], the corresponding Gauss formulae are

$$
\begin{gathered}
\tilde{\nabla}_{X} Y=\nabla_{X} Y+h(X, Y), \\
\tilde{\nabla}_{X}^{*} Y=\nabla_{X}^{*} Y+h^{*}(X, Y),
\end{gathered}
$$

where $h, h^{*}: \Gamma(T M) \times \Gamma(T M) \rightarrow \Gamma\left(T^{\perp} M\right)$ are symmetric and bilinear, called the imbedding curvature tensor (see $[6,8]$ ) of $M$ in $\tilde{M}$ for $\tilde{\nabla}$ and the imbedding curvature tensor of $M$ in $\tilde{M}$ for $\tilde{\nabla}^{*}$, respectively.

In [8], it was also proven that $(\nabla, g)$ and $\left(\nabla^{*}, g\right)$ are dual statistical structures on $M$.

Since $h$ and $h^{*}$ are bilinear, there are linear transformations $A_{\xi}$ and $A_{\xi}^{*}$ on TM defined by

$$
g\left(A_{\xi} X, Y\right)=g(h(X, Y), \xi),
$$




$$
g\left(A_{\widetilde{\zeta}}^{*} X, Y\right)=g\left(h^{*}(X, Y), \xi\right)
$$

for any $\xi \in \Gamma\left(T^{\perp} M\right)$ and $X, Y \in \Gamma(T M)$.

Further (see [8]), the corresponding Weingarten formulae are

$$
\begin{aligned}
& \tilde{\nabla}_{X} \xi=-A_{\xi}^{*} X+\nabla_{X}^{\perp} \xi, \\
& \tilde{\nabla}_{X}^{*} \xi=-A_{\xi} X+\nabla_{X}^{* \perp} \xi,
\end{aligned}
$$

for any $\xi \in \Gamma\left(T^{\perp} M\right)$ and $X \in \Gamma(T M)$. The connections $\nabla^{\perp}$ and $\nabla^{* \perp}$ are Riemannian dual connections with respect to the induced metric on $\Gamma\left(T^{\perp} M\right)$.

Let $\left\{e_{1}, \ldots, e_{n}\right\}$ and $\left\{e_{n+1}, \ldots, e_{m}\right\}$ be orthonormal tangential and normal frames, respectively, on $M$. Then the mean curvature vector fields are defined by

$$
H=\frac{1}{n} \sum_{i=1}^{n} h\left(e_{i}, e_{i}\right)=\frac{1}{n} \sum_{\alpha=n+1}^{m}\left(\sum_{i=1}^{n} h_{i i}^{\alpha}\right) e_{\alpha}, h_{i j}^{\alpha}=g\left(h\left(e_{i}, e_{j}\right), e_{\alpha}\right),
$$

and

$$
H^{*}=\frac{1}{n} \sum_{i=1}^{n} h^{*}\left(e_{i}, e_{i}\right)=\frac{1}{n} \sum_{\alpha=n+1}^{m}\left(\sum_{i=1}^{n} h_{i i}^{* \alpha}\right) e_{\alpha}, h_{i j}^{* \alpha}=g\left(h^{*}\left(e_{i}, e_{j}\right), e_{\alpha}\right),
$$

for $1 \leq i, j \leq n$ and $n+1 \leq \alpha \leq m$.

The Gauss equations for the dual connections $\tilde{\nabla}$ and $\tilde{\nabla}^{*}$, respectively, are given by (see [8])

$$
\begin{aligned}
g(\tilde{R}(X, Y) Z, W)= & g(R(X, Y) Z, W)+g\left(h(X, Z), h^{*}(Y, W)\right)- \\
& -g\left(h^{*}(X, W), h(Y, Z)\right), \\
g\left(\tilde{R}^{*}(X, Y) Z, W\right)= & g\left(R^{*}(X, Y) Z, W\right)+g\left(h^{*}(X, Z), h(Y, W)\right)- \\
& -g\left(h(X, W), h^{*}(Y, Z)\right),
\end{aligned}
$$

Geometric inequalities for statistical submanifolds in statistical manifolds with constant curvature were obtained in [11].

In this section we prove the Chen inequality corresponding to the Chen invariant $\delta(k)$ for statistical submanifolds in statistical manifolds of constant curvature.

We consider an $m$-dimensional statistical manifold $\tilde{M}(\varepsilon)$ of constant curvature $\varepsilon$ and an $n$-dimensional statistical submanifold $M$. Let $p \in M$ and $L$ be a $k$-dimensional subspace of $T_{p} M$. Denote by $\left\{e_{1}, \ldots, e_{k}\right\}$ an orthonormal basis of $L,\left\{e_{1}, \ldots, e_{k}, e_{k+1}, \ldots, e_{n}\right\}$ an orthonormal basis of $T_{p} M$ and $\left\{e_{n+1}, \ldots, e_{m}\right\}$ an orthonormal basis of $T_{p}^{\perp} M$, respectively. 
The Gauss equation implies

$$
\begin{aligned}
\tau= & \frac{1}{2} \sum_{1 \leq i<j \leq n}\left[g\left(R\left(e_{i}, e_{j}\right) e_{j}, e_{i}\right)+g\left(R^{*}\left(e_{i}, e_{j}\right) e_{j}, e_{i}\right)\right]= \\
= & \frac{n(n-1)}{2} \varepsilon+\frac{1}{2} \sum_{1 \leq i<j \leq n}\left[g\left(h^{*}\left(e_{i}, e_{i}\right), h\left(e_{j}, e_{j}\right)\right)+g\left(h\left(e_{i}, e_{i}\right), h^{*}\left(e_{j}, e_{j}\right)\right)-\right. \\
& \left.-2 g\left(h\left(e_{i}, e_{j}\right), h^{*}\left(e_{i}, e_{j}\right)\right)\right]= \\
= & \frac{n(n-1)}{2} \varepsilon+\frac{1}{2} \sum_{r=n+1}^{m} \sum_{1 \leq i<j<n}\left(h_{i i}^{* r} h_{j j}^{r}+h_{i i}^{r} h_{j j}^{* r}-2 h_{i j}^{r} h_{i j}^{* r}\right)= \\
= & \frac{n(n-1)}{2} \varepsilon+\frac{1}{2} \sum_{r=n+1}^{m} \sum_{1 \leq i<j \leq n}\left[\left(h_{i i}^{r}+h_{i i}^{* r}\right)\left(h_{j j}^{r}+h_{j j}^{* r}\right)-h_{i i}^{r} h_{j j}^{r}-h_{i i}^{* r} h_{j j}^{* r}-\right. \\
& \left.-\left(h_{i j}^{r}+h_{i j}^{* r}\right)^{2}+\left(h_{i j}^{r}\right)^{2}+\left(h_{i j}^{* r}\right)^{2}\right]= \\
= & \frac{n(n-1)}{2} \varepsilon+\sum_{r=n+11 \leq i<j \leq n}^{m}\left\{2\left[h_{i i}^{0 r} h_{j j}^{0 r}-\left(h_{i j}^{0 r}\right)^{2}\right]-\frac{1}{2}\left[h_{i i}^{r} h_{j j}^{r}-\left(h_{i j}^{r}\right)^{2}\right]-\right. \\
& \left.-\frac{1}{2}\left[h_{i i}^{* r} h_{j j}^{* r}-\left(h_{i j}^{* r}\right)^{2}\right]\right\},
\end{aligned}
$$

where $h^{0}$ is the second fundamental form of the Riemannian submanifold $M$.

We denote by $\tau_{0}$ the scalar curvature with respect to the Levi-Civita connection and by $\tilde{\tau}_{0}=\sum_{1 \leq i<j \leq n} \tilde{K}_{0}\left(e_{i} \wedge e_{j}\right)$.

The Gauss equation with respect to the Levi-Civita connection gives

$$
\tau_{0}=\tilde{\tau}_{0}+\sum_{r=n+1}^{m} \sum_{1 \leq i<j \leq n}\left[h_{i i}^{0 r} h_{j j}^{0 r}-\left(h_{i j}^{0 r}\right)^{2}\right] .
$$

By substituting Equation (5) into (4), we get

$$
\begin{gathered}
\tau=2\left(\tau_{0}-\tilde{\tau}_{0}\right)+\frac{n(n-1)}{2} \varepsilon-\frac{1}{2} \sum_{r=n+1}^{m} \sum_{1 \leq i<j \leq n}\left[h_{i i}^{r} h_{j j}^{r}-\left(h_{i j}^{r}\right)^{2}\right]- \\
-\frac{1}{2} \sum_{r=n+1}^{m} \sum_{1 \leq i<j \leq n}\left[h_{i i}^{* r} h_{j j}^{* r}-\left(h_{i j}^{* r}\right)^{2}\right] .
\end{gathered}
$$


By using Gauss equation, we have

$$
\begin{aligned}
\tau(L)= & \frac{1}{2} \sum_{1 \leq \alpha<\beta \leq k}\left[g\left(R\left(e_{\alpha}, e_{\beta}\right) e_{\beta}, e_{\alpha}\right)+g\left(R^{*}\left(e_{\alpha}, e_{\beta}\right) e_{\beta}, e_{\alpha}\right)\right]= \\
= & \frac{k(k-1)}{2} \varepsilon+\frac{1}{2} \sum_{1 \leq \alpha<\beta \leq k}\left[g\left(h^{*}\left(e_{\alpha}, e_{\alpha}\right), h\left(e_{\beta}, e_{\beta}\right)\right)+g\left(h\left(e_{\alpha}, e_{\alpha}\right), h^{*}\left(e_{\beta}, e_{\beta}\right)\right)-\right. \\
& \left.-2 g\left(h\left(e_{\alpha}, e_{\beta}\right), h^{*}\left(e_{\alpha}, e_{\beta}\right)\right)\right]= \\
= & \frac{k(k-1)}{2} \varepsilon+\frac{1}{2} \sum_{r=n+1}^{m} \sum_{1 \leq \alpha<\beta \leq k}\left(h_{\alpha \alpha}^{* r} h_{\beta \beta}^{r}+h_{\alpha \alpha}^{r} h_{\beta \beta}^{* r}-2 h_{\alpha \beta}^{r} h_{\alpha \beta}^{* r}\right)= \\
= & \frac{k(k-1)}{2} \varepsilon+\frac{1}{2} \sum_{r=n+1}^{m} \sum_{1 \leq \alpha<\beta \leq k}\left[\left(h_{\alpha \alpha}^{r}+h_{\alpha \alpha}^{* r}\right)\left(h_{\beta \beta}^{r}+h_{\beta \beta}^{* r}\right)-\right. \\
& \left.-h_{\alpha \alpha}^{r} h_{\beta \beta}^{r}-h_{\alpha \alpha}^{* r} h_{\beta \beta}^{* r}-\left(h_{\alpha \beta}^{r}+h_{\alpha \beta}^{* r}\right)^{2}+\left(h_{\alpha \beta}^{r}\right)^{2}+\left(h_{\alpha \beta}^{* r}\right)^{2}\right]= \\
= & \frac{k(k-1)}{2} \varepsilon+\sum_{r=n+1}^{m} \sum_{1 \leq \alpha<\beta \leq k}\left\{2\left[h_{\alpha \alpha}^{0 r} h_{\beta \beta}^{0 r}-\left(h_{\alpha \beta}^{0 r}\right)^{2}\right]-\frac{1}{2}\left[h_{\alpha \alpha}^{r} h_{\beta \beta}^{r}-\left(h_{\alpha \beta}^{r}\right)^{2}\right]-\right. \\
& \left.-\frac{1}{2}\left[h_{\alpha \alpha}^{* r} h_{\beta \beta}^{* r}-\left(h_{\alpha \beta}^{* r}\right)^{2}\right]\right\}= \\
= & 2 \tau_{0}(L)-2 \tilde{\tau}_{0}(L)+\frac{k(k-1)}{2} \mathcal{E}- \\
- & \frac{1}{2} \sum_{r=n+1}^{m} \sum_{1 \leq \alpha<\beta \leq k}\left\{\left[h_{\alpha \alpha}^{r} h_{\beta \beta}^{r}-\left(h_{\alpha \beta}^{r}\right)^{2}\right]+\left[h_{\alpha \alpha}^{* r} h_{\beta \beta}^{* r}-\left(h_{\alpha \beta}^{* r}\right)^{2}\right]\right\} .
\end{aligned}
$$

By subtracting the last equation from (4), we obtain

$$
\begin{aligned}
& (\tau-\tau(L))-2\left(\tau_{0}-\tau_{0}(L)\right) \geq 2\left(\tilde{\tau}_{0}(L)-\tilde{\tau}_{0}\right)+\frac{1}{2}[n(n-1)-k(k-1)] \varepsilon- \\
& \quad-\frac{1}{2} \sum_{r=n+1}^{m}\left(\sum_{1 \leq i<j \leq n} h_{i i}^{r} h_{j j}^{r}-\sum_{1 \leq \alpha<\beta \leq k} h_{\alpha \alpha}^{r} h_{\beta \beta}^{r}\right)- \\
& \quad-\frac{1}{2} \sum_{r=n+1}^{m}\left(\sum_{1 \leq i<j \leq n} h_{i i}^{* r} h_{j j}^{* r}-\sum_{1 \leq \alpha<\beta \leq k} h_{\alpha \alpha}^{* r} h_{\beta \beta}^{* r}\right) .
\end{aligned}
$$

We denote by $\max \tilde{K}_{0}(p)$ the maximum of the Riemannian sectional curvature function of $\tilde{M}(\varepsilon)$ restricted to 2-plane sections of the tangent space $T_{p} M, p \in M$. Obviously

$$
\tilde{\tau}_{0}-\tilde{\tau}_{0}(L) \leq \frac{1}{2}[n(n-1)-k(k-1)] \max \tilde{K}_{0}(p) .
$$

On the other hand, by using Lemma 1, one has

$$
\begin{aligned}
& \sum_{1 \leq i<j \leq n} h_{i i}^{r} h_{j j}^{r}-\sum_{1 \leq \alpha<\beta \leq k} h_{\alpha \alpha}^{r} h_{\beta \beta}^{r} \leq \frac{n-k}{2(n-k+1)}\left(\sum_{i=1}^{n} h_{i i}^{r}\right)^{2}, \\
& \sum_{1 \leq i<j \leq n} h_{i i}^{* r} h_{j j}^{* r}-\sum_{1 \leq \alpha<\beta \leq k} h_{\alpha \alpha}^{* r} h_{\beta \beta}^{* r} \leq \frac{n-k}{2(n-k+1)}\left(\sum_{i=1}^{n} h_{i i}^{* r}\right)^{2} .
\end{aligned}
$$

It follows that

$$
\tau-\tau(L) \geq 2\left(\tau_{0}-\tau_{0}(L)\right)+\frac{1}{2}[n(n-1)-k(k-1)]\left(\varepsilon-2 \max \tilde{K}_{0}(p)\right)-
$$




$$
-\frac{n^{2}(n-k)}{2(n-k+1)}\left[\|H\|^{2}+\left\|H^{*}\right\|^{2}\right] .
$$

We state the following result.

Theorem 2. Let $M$ be an n-dimensional statistical submanifold of an m-dimensional statistical manifold $\tilde{M}(\varepsilon)$ of constant curvature. Then, for any $p \in M$ and any $k$-plane section $L$ of $T_{p} M$, we have:

$$
\begin{aligned}
\tau_{0}-\tau_{0}(L) & \leq \frac{1}{2}(\tau-\tau(L))+\frac{n^{2}(n-k)}{4(n-k+1)}\left[\|H\|^{2}+\left\|H^{*}\right\|^{2}\right]+ \\
+ & \frac{1}{2}[n(n-1)-k(k-1)]\left(\max \tilde{K}_{0}(p)-\frac{\varepsilon}{2}\right) .
\end{aligned}
$$

Moreover, the equality holds at a point $p \in M$ if and only if there exist orthonormal bases $\left\{e_{1}, \ldots, e_{n}\right\}$ of $T_{p} M$ and $\left\{e_{n+1}, \ldots, e_{m}\right\}$ of $T_{p}^{\perp} M$ such that

$$
\left\{\begin{array}{l}
\sum_{\alpha=1}^{k} h_{\alpha \alpha}^{r}=h_{j j}^{r}, \forall j \in\{k+1, \ldots, n\}, \\
\sum_{\alpha=1}^{k} h_{\alpha \alpha}^{* r}=h_{j j}^{* r}, \forall j \in\{k+1, \ldots, n\}, \\
h_{i j}^{r}=h_{i j}^{* r}=0, \forall 1 \leq i<j \leq n,(i, j) \notin\{1, \ldots, k\}^{2},
\end{array}\right.
$$

for any $r \in\{n+1, \ldots, m\}$.

Author Contributions: Conceptualization, I.M. and R.-I.M.; methodology, I.M.; validation, I.M.and R.-I.M.; formal analysis, R.-I.M.; investigation, I.M. and R.-I.M.; resources, R.-I.M.; writing-original draft preparation, I.M.; writing-review and editing, R.-I.M.; visualization, R.-I.M.; supervision, I.M.; project administration, I.M. All authors have read and agreed to the published version of the manuscript.

Funding: This research received no external funding.

Institutional Review Board Statement: Not applicable.

Informed Consent Statement: Not applicable.

Data Availability Statement: Not applicable.

Conflicts of Interest: The authors declare no conflict of interest.

\section{References}

1. Chen, B.-Y. Some pinching and classification theorems for minimal submanifolds. Arch. Math. 1993, 60, 568-578. [CrossRef]

2. Chen, B.-Y. Some new obstructions to minimal and Lagrangian isometric immersions. Jpn. J. Math. 2000, 26, 105-127. [CrossRef]

3. Mihai, I.; Mihai, R.I. An algebraic inequality with applications to certain Chen inequalities. Axioms 2021, 10, 7. [CrossRef]

4. Chen, B.-Y. Geometry of Submanifolds; M. Dekker: New York, NY, USA, 1973.

5. Chen, B.-Y. Relations between Ricci curvature and shape operator for submanifolds with arbitrary codimensions. Glasg. Math. J. 1999, 41, 33-41. [CrossRef]

6. Amari, S. Differential-Geometrical Methods in Statistics; Springer: Berlin, Germany, 1985.

7. Shima, H. The Geometry of Hessian Structures; World Scientific: Singapore, 2007.

8. Vos, P.W. Fundamental equations for statistical submanifolds with applications to the Bartlett correction. Ann. Inst. Statist. Math. 1989, 41, 429-450. [CrossRef]

9. Dillen, F.; Nomizu, K.; Vrancken, L. Conjugate connections and Radon's theorem in affine differential geometry. Monatsh. Math. 1990, 109, 221-235. [CrossRef]

10. Opozda, B. Bochner's technique for statistical structures. Ann. Glob. Anal. Geom. 2015, 48, 357-395. [CrossRef]

11. Aydin, M.E.; Mihai, A.; Mihai, I. Some inequalities on submanifolds in statistical manifolds of constant curvature. Filomat 2015, 29, 465-477. [CrossRef] 